\title{
Critical limb ischemia caused by adductor canal compression syndrome - case report with the review of the literature
}

\author{
Piotr Kaszczewski', Tomasz Ostrowski', Jarosław Żyłkowski², Zbigniew Gałązka' \\ 'Department of General and Endocrine Surgery, Medical University of Warsaw, Poland \\ ${ }^{2} 2^{\text {nd }}$ Division of Radiology, Medical University of Warsaw, Poland
}

\begin{abstract}
We report a case of a 55-year-old male who was diagnosed with the adductor canal compression syndrome - a rarely described nontraumatic disorder of the lower limb resulting from the external compression of the superficial femoral artery in the adductor canal, that may present with the symptoms ranging from intermittent exercise-induced claudication up to arterial obstruction and critical limb ischemia.
\end{abstract}

Key words: adductor canal compression syndrome, critical limb ischaemia, superficial femoral artery occlusion, nontraumatic vascular disorder, arterial compression

Acta Angiol 2018; 24, I: 14-17

\section{Case report}

A 55-year old Caucasian male was admitted to our department with the diagnosis of the critical left lower limb ischemia. The patient presented a sudden onset of exercise-induced claudication 3 months preceding hospitalization. Since then the symptoms were perpetually progressing, eventually resulting in rest pain. Any lower extremity trauma, intake of anabolic agents or other chemical substances including drugs was strongly denied. No history of chronic diseases, alcohol abuse or intake of any medications was recorded. The only risk factor for atherosclerosis was smoking habit - about 20 cigarettes per day for about 30 years ( 30 pack years). The patient had a deep vein thrombosis excluded with a Doppler-Duplex examination and because of the constant exacerbation of symptoms referred to vascular surgery department. On admission, the patient complained of rest pain, which was preceded by intermittent claudication (claudication distance below 10 meters), paresthesia and coldness of the limb. There was palpable pulse on left femoral artery and absent pulses in popliteal, posterior tibial and dorsalis pedis artery.
A CT-scan revealed patent common femoral, deep femoral and proximal part of superficial femoral artery and $1 \mathrm{l} \mathrm{cm}$ occlusion of the distal part of the superficial femoral artery from the level of the hiatus of the adductor canal with the balloting thrombus below the occluded part of the artery and patent popliteal artery supplied from the collateral circulation (Fig. I). There were no hemodynamically significant atherosclerotic lesions in the lower extremity arteries. The patient was qualified for surgical treatment. From the medial approach at the level of the Hunter canal hiatus, a thrombectomy was performed. During surgical exploration of the adductor canal a fibromuscular band, arising from the adductor magnus muscle, overlying, running across and compressing the superficial femoral artery was exposed on the canal outlet (Fig. 2). The fibromuscular band was subsequently excised, a thrombectomy was successfully performed (Fig. 3) and the artery was closed with the use of the vein interposition patch, harvested from the distal part of the ipsilateral saphenous vein. The patient was discharged from hospital in the 5 days from the surgery with a palpable pulse in femoral, popliteal, anterior and posterior tibial arteries, and the proper three 

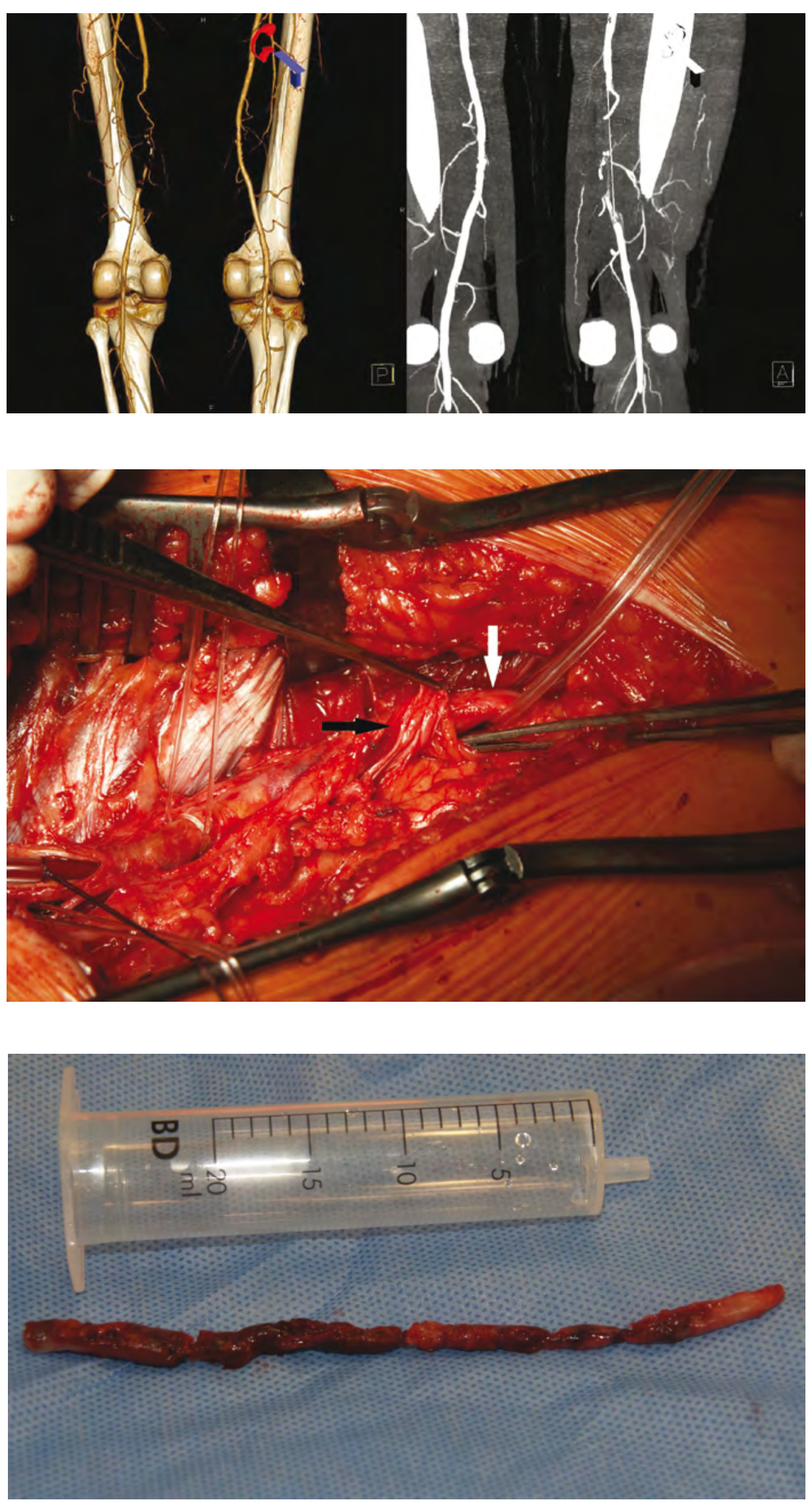

Figure I. A - three dimensional reconstruction demonstrates occlusion of the superficial femoral artery - posterior view; $\mathrm{B}-\mathrm{a}$ computed tomography image of the II $\mathrm{cm}$ occlusion of the artery in question - anterior view

Figure 2. The fibromuscular band ( black arrow) — arising from the adductor magnus muscle, overlying, running across and compressing the superficial femoral artery (white arrow)

Figure 3. The thrombotic material removed from the superficial femoral artery 


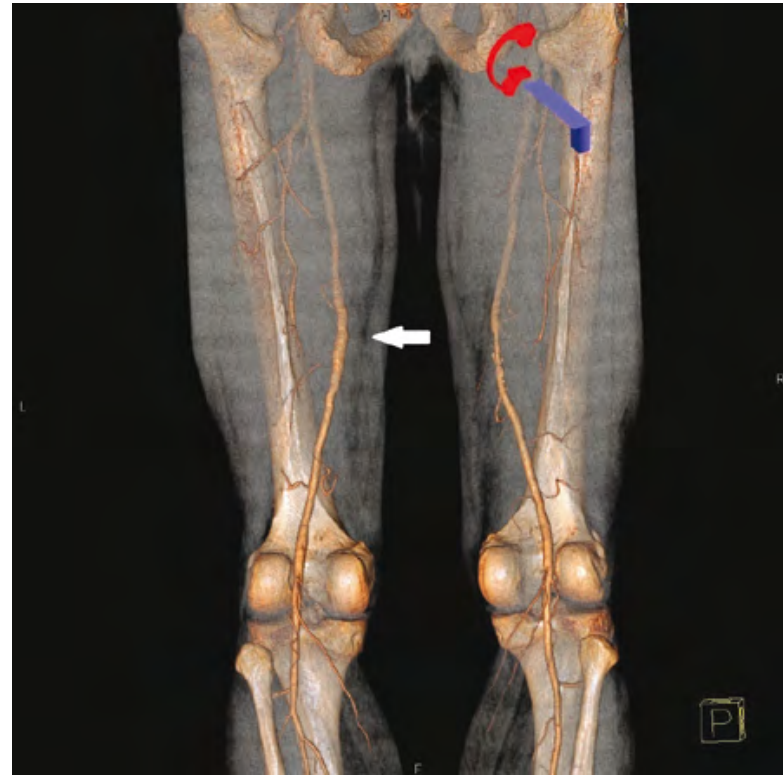

Figure 4. A 3 dimensional reconstruction a follow-up computed tomography angiography 6 months after the surgery demonstrates patency and excellent result of the surgical treatment of the right superficial femoral artery (white arrow) - posterior view

phasic waveforms in Doppler-Duplex examination in femoral, popliteal and calf arteries. A follow-up CT scan 6 months after the operation confirmed a very good result of the treatment (Fig. 4). The patient reported no symptoms of adductor canal compression syndrome.

\section{Discussion}

The adductor canal compression syndrome is a rare nontraumatic disorder of the lower limb in which an external compression of the neurovascular structures in the Hunter canal may result either in neurological or vascular symptoms including acute thrombosis leading to critical lower limb ischemia [I-3]. It was first described in 1950 by Palma [4]. Clinical symptoms include exercise-induced claudication, paresthesias and muscle weakness in the limb resulting with impaired performance and are usually chronic and progressive. However, more severe clinical course as the consequence of acute thrombosis or dissection of the artery might occur [5]. This scenario took place in our patient, who previously complained of exercise-induced intermittent claudication and developed a critical limb ischemia in three months from the onset of the symptoms.

While previously Palma thought the disease not to affect female because of the more oblique femur and larger adductor canal, currently the case reports describing adductor canal compression syndrome both in male and female patients are published $[2,6]$.

Neurological symptoms may be a result of saphenous nerve compression at the level of the hiatus of the canal causing pain of the medial part of the knee - in the area of saphenous nerve sensory innervation and are not connected with any vascular compression $[7,8]$. The compression of the superficial femoral artery leads usually to exercise-induced claudication but may also be the cause of thrombosis and critical limb ischemia connected with the risk of the limb loss. The compression may be caused by either hypertrophied muscles forming the adductor canal: adductor magnus and vastus medialis at the level of adductor hiatus or by abnormal fibrous or fibromuscular band like structure arising from adductor magnus [5, 9]. The latter relates to the occlusion of the superficial femoral artery, which was also the case in our patient [3].

Non-traumatic vascular injuries of the lower limb such as: intimal hyperplasia, stenosis or kinking of iliac arteries inflexion, adductor canal syndrome or popliteal entrapment syndrome, effort induced venous thrombosis or chronic exertional compartment syndrome is a group of disorders affecting mainly young and healthy athletes, often trained professionals competing at the professional level without concomitant diseases and cardiovascular risk factors [9]. Among the group of the mentioned disorders, the adductor canal compression syndrome is most frequent in skiers and runners [10]. Our patient was not a professional sportsman but as a blue-collar worker, he is used to lifting huge amount of load, up to even few tons per day, for the last 30 years.

The patient was 55-years-old when the symptoms developed, which is in consistency with the mean age of the onset of the symptoms of the adductor canal compression syndrome in cases described in the literature, which is $45 \pm 1 \mathrm{I}$ [8]. The factor connected with the age of the patient, that may facilitate the compression syndrome to develop, is the age-related modification of connective tissue of the adductor canal resulting in stronger adherence of the connective tissue to the adjacent structures and the consecutive loss of the sliding movement of the vessels during activity [II].

The patients with the sudden onset of the claudication, especially those without previous history and the risk factors of the cardiovascular diseases should have a Color Duplex Doppler examination and a CT scan performed. The MRI examination, providing information about soft tissues should be also considered. These examinations lead to proper diagnosis and differentiate the adductor canal compression syndrome from popliteal entrapment syndrome, which can present similar clinical course $[3,5,6,8,10]$.

Surgical excision of the abnormal fibromuscular band and the angioplasty or bypass of the affected arterial segment with or without the use of venous patch or interposition graft is the treatment of choice. In acute arterial occlusion, a catheter-directed thrombolysis followed by surgical operation may be performed $[5,6,8]$. 


\section{Conflict of interest}

None.

\section{References:}

I. Verta MJ, Vitello J, Fuller J. Adductor canal compression syndrome. Arch Surg. 1984; 1 19(3): 345-346, indexed in Pubmed: 6696630.

2. Porr J, Chrobak K, Muir B. Entrapment of the saphenous nerve at the adductor canal affecting the infrapatellar branch - a report on two cases. J Can Chiropr Assoc. 2013; 57(4): 341-349, indexed in Pubmed: 24302782.

3. Ehsan O, Darwish A, Edmundson C, et al. Non-traumatic lower limb vascular complications in endurance athletes. Review of literature. Eur J Vasc Endovasc Surg. 2004; 28(I): I-8, doi: 10.1016/j.ejvs.2004.02.002, indexed in Pubmed: 15177226 .

4. Palma EC. Stenosing arteriopathies of the lower extremity; Hunter's canal and third abductor ring syndrome. Bol Trab Acad Argent Cir. 1950; 34(21): 77I-787, indexed in Pubmed: 14791668.

5. Perlowski AA, Jaff MR. Vascular disorders in athletes. Vasc Med. 2010; 15(6): 469-479, doi: 10.1177//358863X10382944, indexed in Pubmed: 20884798.
6. Walensi M, Berg C, Piotrowski M, et al. Adductor Canal Compression Syndrome in a 46-Year-Old Female Patient Leading to Acute External lliac, Femoral, and Popliteal Artery Thrombosis and Critical Ischemia: A Case Report. Ann Vasc Surg. 2017; 38: 319.ell-319.el5, doi: 10.1016/j.avsg.2016.05.134, indexed in Pubmed: 27554690.

7. Morganti CM, McFarland EG, Cosgarea AJ. Saphenous neuritis: a poorly understood cause of medial knee pain. J Am Acad Orthop Surg. 2002; 10(2): 130-137, indexed in Pubmed: II 929207.

8. Sapienza P, Tartaglia E, Venturini L, et al. Adductor canal compression syndrome: a forgotten disease. Ann Ital Chir. 2014; 85(ePub), indexed in Pubmed: 25559676.

9. Mosley JG. Arterial problems in athletes. Br J Surg. 2003; 90(12): |46|-|469, doi: 10.1002/bjs.4374, indexed in Pubmed: |4648723.

10. Casey E, Lento P, Ihm J, et al. Vascular Injuries in the Lower Limb of Athletes. Nerve and Vascular Injuries in Sports Medicine. 2009: 183-198, doi: 10.1007/978-0-387-76600-3_13.

II. de Oliveira F, de Vasconcellos Fontes RB, da Silva Baptista J, et al. The connective tissue of the adductor canal - a morphological study in fetal and adult specimens. J Anat. 2009; 214(3): 388-395, doi: 10.1 III/j.1469-7580.2009.01047.x, indexed in Pubmed: 19245505. 